\title{
Muscle Endurance Testing Using an Isokinetic Dynamometer and A Recumbent Stepper: A Correlation Study
}

\author{
Pepin, Marie-Eve ${ }^{*}$, Thornton Alyssa ${ }^{1}$; Gasperoni Vittoria ${ }^{1}$; Kosky Jillian ${ }^{1}$, Lowrey Laura ${ }^{1}$, Varghese Ansu ${ }^{1}$ \& Galen \\ Sujay $^{2}$ \\ ${ }^{1}$ Physical Therapy Program, Wayne State University, Detroit, MI, United States. \\ ${ }^{2}$ Department of Physical Therapy, Georgia State University, Atlanta, GA, United States.
}

Article Details
Article Type: Research Article
Received date: $11^{\text {th } J u n e, ~} 2021$
Accepted date: $12^{\text {th }}$ July, 2021
Published date: $14^{\text {th }}$ July, 2021

"Corresponding Author: Pepin, Marie-Eve, PT, DPT, MSPT, OMPT, Assistant Professor, Department of Physical Therapy, Wayne State University, 259 Mack Avenue, Detroit, MI 48201, United States. E-mail: marie.eve.pepin@wayne.edu

Citation: Marie-Eve, P., Alyssa, T., Vittoria,G., Jillian,K., Laura, L., Ansu,V., \& Sujay, G., (2021). Muscle Endurance Testing Using An Isokinetic Dynamometer And A Novel Recumbent Stepper: A Correlation Study. J Rehab Pract Res, $2(2): 121$. https://doi.org/10.33790/jrpr1100121

Copyright: (C2021, This is an open-access article distributed under the terms of the Creative Commons Attribution License 4.0, which permits unrestricted use, distribution, and reproduction in any medium, provided the original author and source are credited.

\begin{abstract}
s
Purpose: A lack of objective and validated clinical measures to assess Quadriceps muscle endurance (ME) currently exists. The Transitt model of the NuStep recumbent stepper has the capability to measure ME but has not been validated. Therefore, the aim of this study was to investigate the association between ME measures on the NuStep and the isokinetic dynamometer (ID).
\end{abstract}

Methods: 30 healthy individuals were recruited to participate. On both the NuStep $\left(70^{\circ} / 90^{\circ}\right.$ knee flexion) and ID $\left(70^{\circ}\right.$ knee flexion), the participants were instructed to hold a submaximal isometric unilateral knee extension contraction until exhaustion. Descriptive statistics, inferential statistics and correlation analyses were used with significance set at $\mathrm{p}<0.05$.

Results: There was excellent correlation ( $\mathrm{r}$ between 0.813 to 0.840 , $\mathrm{p}<0.001)$ between the average force generated by the individuals on the ID and the NuStep. There was a fair correlation ( $\mathrm{r}$ between 0.331 to $0.413, \mathrm{p}<0.05$ ) for the ME measures between the ID and the NuStep.

Conclusions: The NuStep shows excellent agreement for average force measurements. Future studies are needed to validate the NuStep as an objective measure of ME in both healthy and clinical populations.

Keywords: Endurance testing, Isometric contraction, quadriceps, recumbent stepper, COPD, Isokinetic

\section{Introduction}

Muscle strength and endurance are key components in formulating an exercise prescription in physical therapy. Muscular strength refers to the "maximal force (measured in newtons or pounds) that can be generated by a specific muscle or muscle group" while endurance refers to "the ability of a muscle group to perform repeated contractions over a period of time sufficient to cause muscular fatigue, or to maintain a specific percentage of maximum voluntary contraction for a prolonged period of time" [1]. The benefits of muscle strength training/ testing is well documented. It is also important to objectively assess muscle endurance (ME) because it has been correlated with balance [2], fall risks [3], cardiovascular endurance, body fat, functional ability and even mortality [4].

Individuals with Congestive Heart Failure (CHF) [5], patellofemoral pain [6] or knee osteoarthritis [7] may have a reduction in quadriceps $\mathrm{ME}$ or a susceptibility to premature fatigability and force generation capability. Evidence indicates that individuals with chronic obstructive pulmonary disease (COPD) are especially at risk for decreased ME [8-11]. Decreased ME has been correlated with lower physical activity and functional performance in individuals with COPD [12]. Furthermore, studies have found that a quadriceps shift from type I (oxidative) muscle fibers to type II (glycolytic) fibers was related to lung function and mortality in patients with COPD, and quadriceps endurance have been related to severity of lung obstruction by some authors [8]. These findings support ME as an important clinical measure for a variety of patients.

There are a variety of protocols for quantifying lower extremity ME, including repeated squats, wall sits and protocols using an isokinetic dynamometer (ID). Except for the ID, none of these clinical measures of endurance have been validated. The ID is commonly used for measuring endurance in clinical trials or in research settings and may be considered the gold standard for measuring ME in a laboratory. The ID protocols are however often difficult to implement as a routine assessment in a clinical population and the ID tests muscles in non-functional positions. The recumbent stepper (NuStep, Inc., Ann Arbor, MI) is an exercise modality that is widely used in numerous rehabilitation settings and can be used by individuals of differing fitness levels. The Transitt model of the NuStep recumbent stepper has the capability to be used as a dynamometer to assess muscle endurance and force generation capability. Indeed, it can reproduce the underpinning construct of a ME test (i.e. to generate a sub-maximal muscle force and sustain it over a period of time), despite requiring a different set up and patient positioning than the ID. However, it is not clear how force generation/ME measures recorded by the NuStep relate to those recorded by the ID, and there has been no study to date that has investigated the association between these two measures. 
The purpose of this pilot study was therefore to investigate the association between muscle endurance measures recorded by the NuStep and the ID in healthy individuals. The authors hypothesized that there would be good agreement between the NuStep and ID when measuring ME in healthy individuals.

\section{Materials And Methods \\ Design}

The study was performed at Wayne State University. It was a single visit, cross-sectional correlational study using two different devices (NuStep and ID) to measure lower extremity ME.

\section{Participants}

Healthy participants between the ages of 18 to 80 were recruited using flyers and word of mouth. Exclusion criteria included any diagnosed neurological, orthopedic, or cardiopulmonary disease that would limit the individual's ability to perform maximal voluntary knee extension contractions (MVC).

\section{Procedures}

Approval by the institutional board of Wayne State University was obtained and participants gave their informed consent prior to the start of the study. Data collectors received extensive training and practice on the use of the NuStep and ID. Demographics collected included age, gender, height and weight. The subjects were electronically randomized for the following: 1) type of device on which endurance was tested first (NuStep or ID), 2) lower extremity to be tested first (left or right) and 3) initial knee flexion angle to be tested on the NuStep (70 or 90 degrees). A 15-minute rest break was given between testing on the ID and NuStep.

\section{Humac Isokinetic Dynamometer (ID)}

The ID (Humac Norm, CSM Inc., Stoughton, MA) was calibrated in accordance to the manufacturer's specification. Participants were positioned with their upper body strapped firmly against the backrest of the chair. Hips were positioned at $90^{\circ}$ of flexion and knees at $70^{\circ}$ of knee flexion to maintain a more optimal length tension relationship of the quadriceps muscles. Straps were tightened across the subject's chest, hips, thighs and ankle (Figure 1).

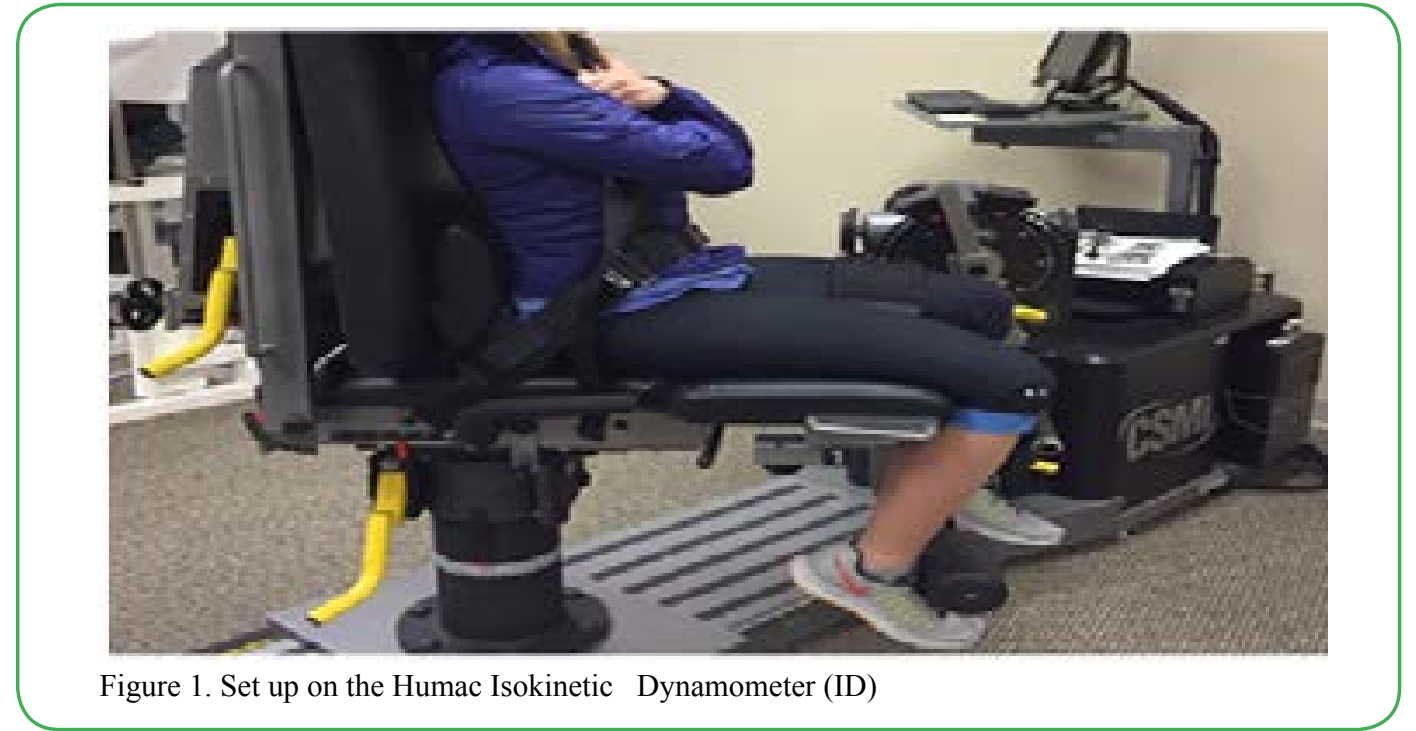

The endurance testing protocol used for this study was similar to that described by Thornley et al. [13]. First, maximal quadriceps contraction force was measured by having the participants perform three maximal unilateral knee extension holds for five-seconds each, with a 30 -second rest break in between each repetition. The highest recorded force from the three attempts was used to calculate the target force range $(80-90 \%$ of the maximal force). After the maximal contraction force was tested, the participants rested for 2 minutes before performing the endurance test. The participant held an isometric knee extension contraction with a force between
$80-90 \%$ of maximal contraction for as long as possible. The test was terminated if the subject dropped below the $80 \%$ of the maximal contraction for greater than five seconds. The test was repeated on the other leg. Standardized verbal instructions were used by the tester, which they read off a printed protocol sheet for consistency. Endurance was calculated using the area under the force/time curve (Figure 2). This is illustrated by the shaded area in Figure 2. The Thoracic Society/European Respiratory Society recommends measuring time to task failure over fatigability for measuring muscle endurance [10].

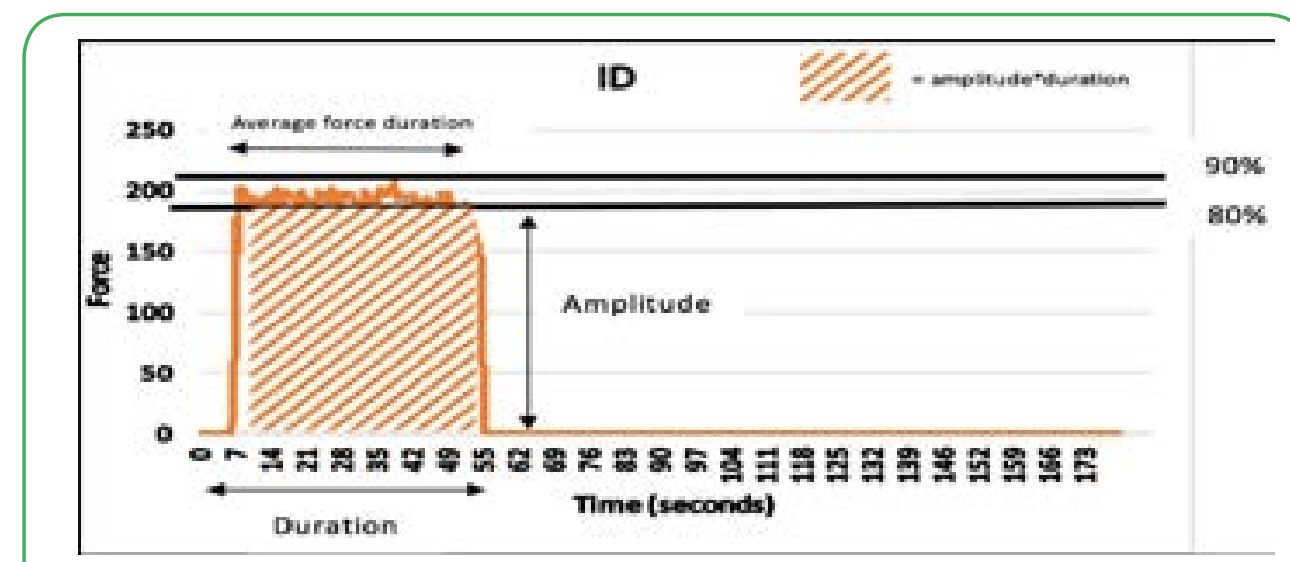

Figure 2: Endurance Calculation (Force*Duration) 


\section{NuStep}

The instrumented "NuStep Transit", a model of Nu-Step recumbent stepper was used for this study. ME was tested at $70^{\circ}$ and $90^{\circ}$ of knee flexion. The $90^{\circ}$ position was advised by the manufacturer while the $70^{\circ}$ was chosen to resemble the ID test position (Figure 3). A single researcher measured all knee flexion angles with a goniometer for consistency. The participants sat on the NuStep secured with a safety lap belt; a foot strap secured the foot onto the foot plate. The nontested lower extremity was placed on a foot stool so that the muscles would be minimally recruited during testing (Figure 3).

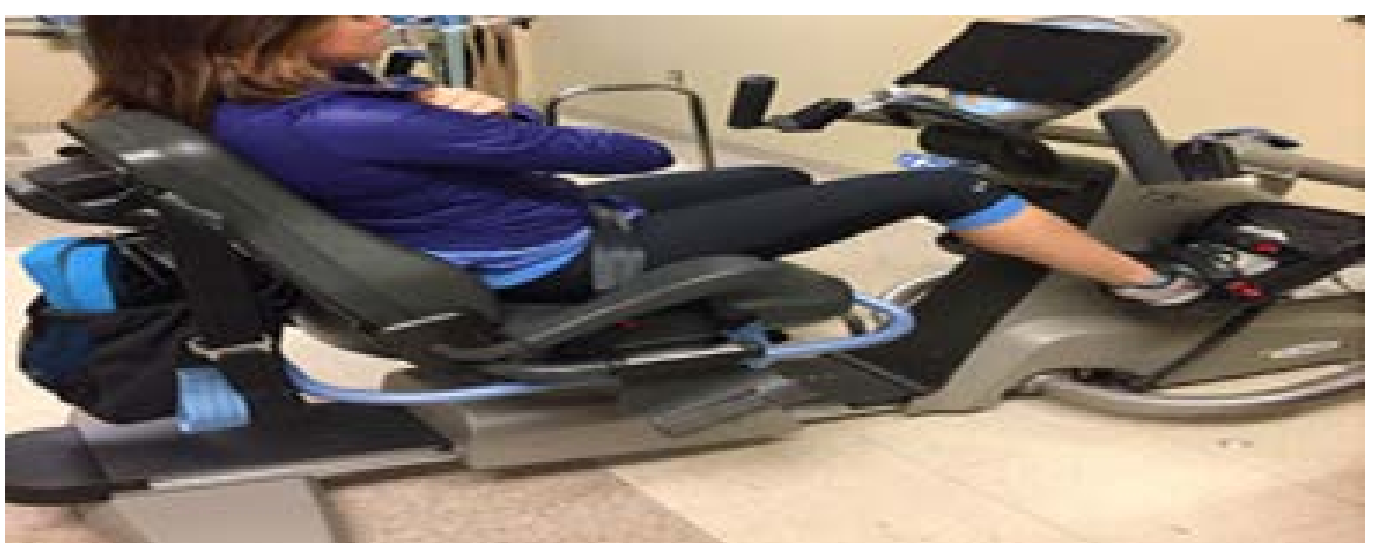

Figure 3. Set up on the NuStep recumbent stepper

The participants were instructed to hold a submaximal isometric unilateral knee extension contraction equal to $50 \%$ of bodyweight (manufacture specification), for as long as possible up to a maximum of five-minutes. The participants were able to receive visual feedback regarding the amount of force produced by looking at a tablet screen connected to the NuStep. The test was repeated on the opposite lower extremity at the same knee flexion angle and with the same standardized procedure. Participants then received a minimum of a two-minute rest period prior to the tests being repeated, at the remaining knee flexion angle $\left(70^{\circ}\right.$ or $\left.90^{\circ}\right)$. Consistent verbal commands were used, and encouragement was given at one-minute time intervals during testing. Like the ID, endurance was calculated using the area under the force/time curve (figure 2).

\section{Statistical analysis}

A convenience sample of 30 healthy participants was deemed adequate for this pilot study, because there were no previous data available to perform a sample size calculation. Statistical analysis was carried out using SPSS version 24.0 (SPSS, Inc. Chicago, IL). Data was tested for normality using the Shapiro-Wilks test. Descriptive statistics (means, standard deviations [SDs]) were calculated for all outcome measures. Inferential statistics were used to examine for differences between endurance measures at $70^{\circ}$ and $90^{\circ}$ of knee flexion on the NuStep. Correlation analysis examined the relationship between NuStep and ID measures. The strength of the correlation/ association between the two measures of ME was assessed based on criteria provided by Portney and Watkins, which is as follows: a correlation from 0.00 to 0.25 indicates small or no relationship; between 0.25 and 0.50 , a fair relationship; between 0.50 and 0.75 , a moderate to good relationship; above 0.75 , excellent relationship [14]. All statistical tests were performed with significance set at $\mathrm{p}<0.05$.

\section{Results}

Thirty individuals (men=13, women=17) with an average age of 24.37 (SD: 1.19) years participated in the study. All participants were able to sustain the isometric contraction for a longer duration (113.43 to 202.23 seconds) and produce a higher force output ( 374.10 to $376.77 \mathrm{~N}$ ) on the NuStep (Table 1) compared to the ID. Means, standard deviations (SD) and confidence intervals (IC) for all reported variables are presented in Table 1 .

Although the force recorded were not the same magnitude between the NuStep and ID, there were no proportional errors recorded and the force measures on the two devices related well to each other as depicted by the Bland and Altman plots (figure 4).

\begin{tabular}{|c|c|c|c|c|c|c|c|c|c|}
\hline & \multicolumn{3}{|c|}{ NuStep 70 degrees } & \multicolumn{3}{|c|}{ NuStep 90 degrees } & \multicolumn{3}{|l|}{ ID } \\
\hline & Mean & SD & IC & Mean & SD & $\mathrm{IC}$ & Mean & SD & IC \\
\hline Right Duration (S) & 202.23 & 87.81 & $169.45,235.02$ & 113.43 & 68.56 & $87.83,139.03$ & 32.98 & 18.84 & $25.94,40.02$ \\
\hline Left Duration (S) & 217.47 & 116.01 & $174.15,260.79$ & 97.47 & 51.21 & $78.35,116.59$ & 34.87 & 15.42 & $29.10,40.63$ \\
\hline Right Force (N) & 376.77 & 57.48 & $355.30,398.23$ & 374.10 & 57.03 & $352.80,395.39$ & 140.61 & 42.03 & $124.91,156.30$ \\
\hline Left Force $(\mathrm{N})$ & 375.58 & 58.47 & $353.75,397.41$ & $373 . .80$ & 58.58 & $351.92,395.67$ & 137.56 & 43.51 & $121.32,153.81$ \\
\hline $\begin{array}{l}\text { Right Force } \\
\text { Duration (NS) x } \\
104\end{array}$ & 7.60 & 3.38 & $8.87,6.34$ & 4.26 & 2.73 & $5.28,3.24$ & 0.62 & 0.38 & $0.76,0.48$ \\
\hline $\begin{array}{l}\text { Left Force } \\
\text { Duration (NS) x } \\
104\end{array}$ & 8.31 & 4.77 & $10.09,6.53$ & 3.68 & 2.09 & $4.47,2.90$ & 0.62 & 0.32 & $0.74,0.51$ \\
\hline
\end{tabular}




\section{Right ID vs Right NuStep 70}

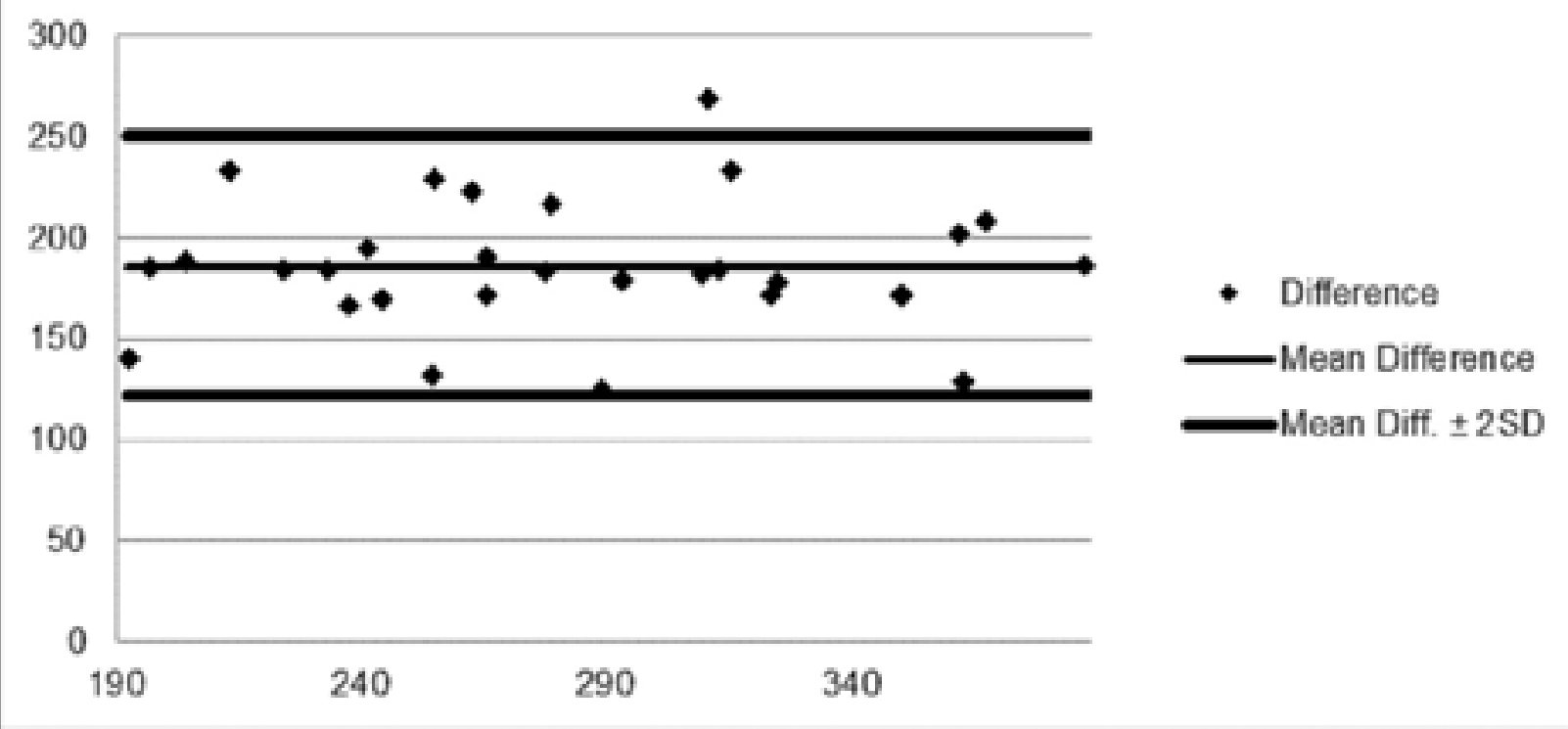

Figure 4: Bland and Altman plots showing the relationship between force measurements on the ID compared to the Nu-Step

There was excellent correlation between the mean force generated by the individuals on the ID and the NuStep at each knee flexion angle for both lower extremities ( $\mathrm{r}$ between 0.813 to $0.840, \mathrm{p}<0.001$ ) (Table 2). There was a fair correlation between the endurance measures (area under the curve of force duration measures) recorded by the ID and the NuStep for both lower extremities ( $\mathrm{r}$ between 0.331 to $0.413, \mathrm{p}<0.05)$ (Table 3). Many of the participants $(\mathrm{n}=13)$ held the isometric knee extension contraction for the full five-minutes when the participants were placed in $70^{\circ}$ of knee flexion, and therefore a ceiling effect was observed (Figure 5a). This ceiling effect was not seen when the participant performed the same test at $90^{\circ}$ of knee flexion. (Figure $5 b$ ).

\begin{tabular}{|c|c|c|c|c|}
\hline & $\begin{array}{l}\text { NuStep Right } \\
70\end{array}$ & $\begin{array}{l}\text { NuStep Right } \\
90\end{array}$ & NuStep Left 70 & NuStep Left 90 \\
\hline Right ID & $\begin{array}{l}\mathrm{R}=0.820 \\
\mathrm{p}<0.001\end{array}$ & $\begin{array}{l}\mathrm{R}=0.813, \\
\mathrm{p}<0.001\end{array}$ & & \\
\hline Left ID & & & $\begin{array}{l}\mathrm{R}=0.816, \\
\mathrm{p}<0.001\end{array}$ & $\begin{array}{l}\mathrm{R}=0.840, \\
\mathrm{p}<0.001\end{array}$ \\
\hline
\end{tabular}

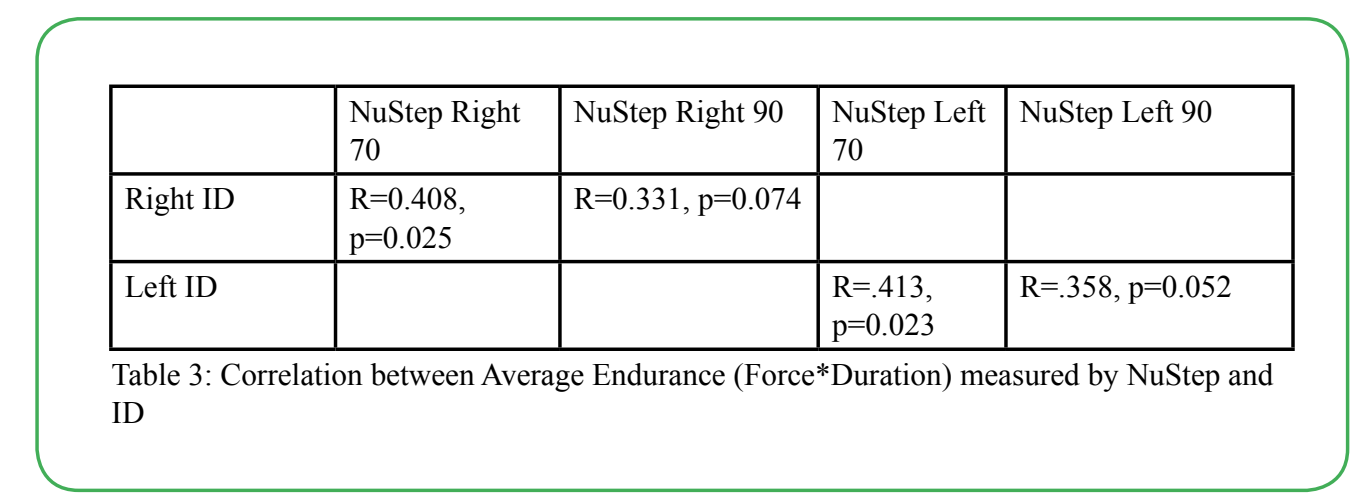




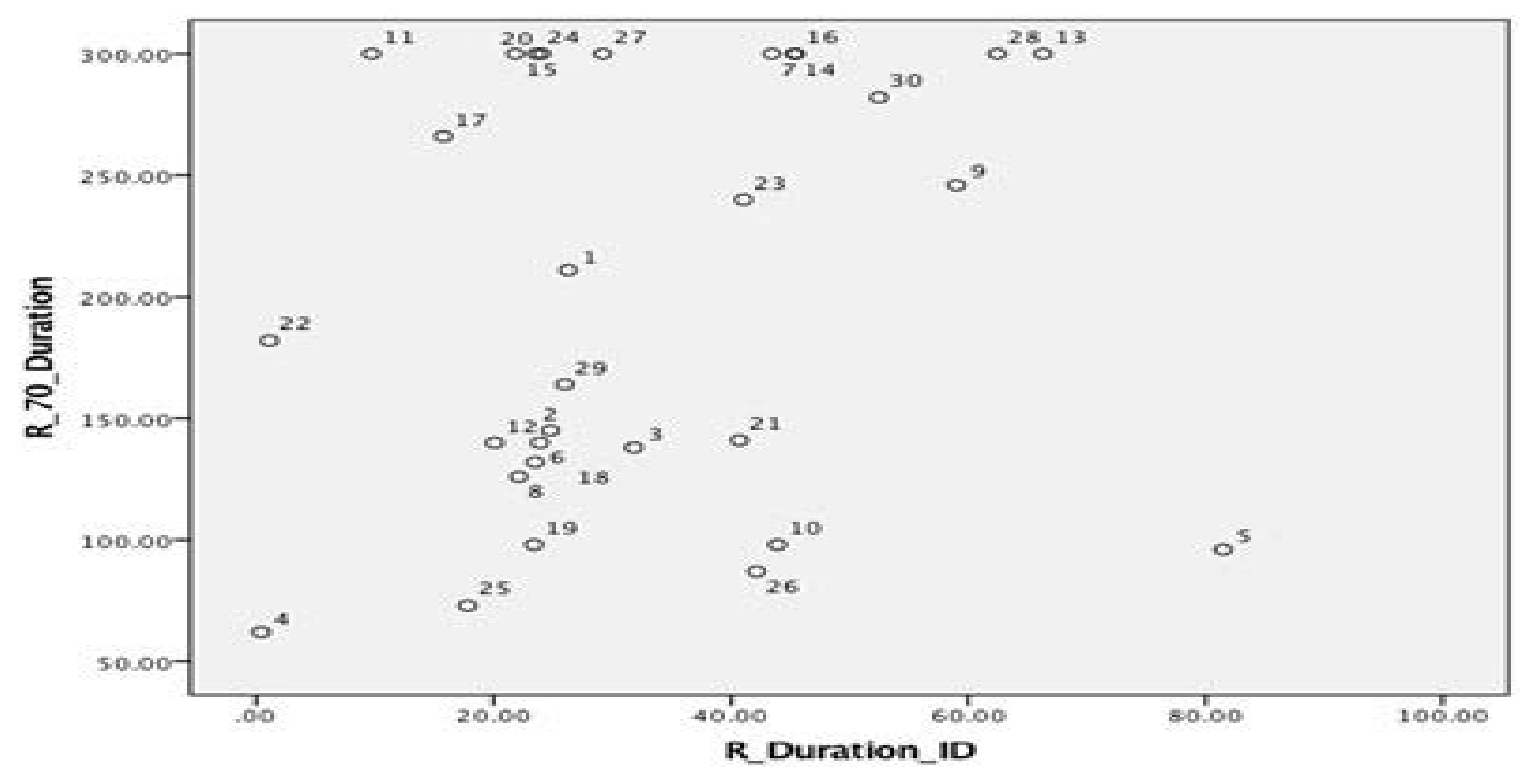

Figure 5: Duration of contraction on the NuStep on the $\mathrm{Y}$ axis and ID on the $\mathrm{X}$ axis. Data in figure " $\mathrm{A}$ " were measured at $70^{\circ}$ knee flexion while $\mathrm{B}$ at $90^{\circ}$ knee flexion

\section{Disussion}

The purpose of this pilot study was to investigate the association between muscle endurance (ME) measures recorded by the NuStep and the ID in healthy individuals. As explained earlier, although both modalities have the capability to test lower extremity ME, the positioning of the individual for the test, and action performed (knee extension), are not entirely identical. Therefore, an analysis of association was performed, and the authors had hypothesized that there would be a good agreement between the two measures.

There was a fair correlation between the ME measures recorded by the NuStep and the ID among the study participants. This is the first study correlating endurance measures on the NuStep and ID. Several reasons might explain this fair correlation. First and foremost, the lower extremity movement performed on the ID and NuStep were different. The ID required an open chain movement while the NuStep movement resembled more of a closed chain. It is therefore very likely that muscles other than the quadriceps were recruited on the NuStep and that the muscles would have fatigued differently. Second, the endurance measures on the NuStep lasted longer therefore may have required greater motivation and more persistent levels of concentration than on the ID, which could have accounted for some of the differences observed. Thirdly, the protocols for measuring endurance were different. The subjects had to maintain a force equal to $50 \%$ body weight on the NuStep compared to a force equal to $80 \%$ $90 \%$ knee extension MVC on the ID. This is an important difference as a lighter subject would have had to hold a smaller force on the NuStep, irrespective of his/her maximal strength. This a limitation of the endurance protocol which cannot be manually altered on the NuStep. Finally, the ID was used as a gold standard because it is the most commonly used device to measure quadriceps endurance in the literature. However, protocols described in the literature vary widely $[15,16]$ and a standard protocol has yet to be established and validated [17]. It is noteworthy that the authors did attempt to use the protocol described by White and et al. White et al. [18] used 3 sets of 12 maximum isometric knee extension contractions spaced by 5 second rest and measured a fatigue index using the performance on the $1^{\text {st }}$ and $12^{\text {th }}$ repetition. Upon preliminary testing, it was observed that there was no decrease in force output over time and the protocol was therefore not deemed sensitive enough to detect fatigue. Therefore, a modified version of Thorley's protocol [13], as described in the method, was used for this study.

The investigators had decided on a termination criterion of 5 minute on the NuStep to avoid lengthy testing which would be impractical in the clinic. A ceiling effect was observed when several subjects reached this termination criteria when tested at $70^{\circ}$ knee flexion (Figure 4a) indicating that several participants had not fatigued enough to dip below the $50 \%$ threshold at the completion of the test. The investigators therefore recommend using the $90^{\circ}$ knee flexion angle on the NuStep in the future.

In contrast to the fair correlation, there was excellent correlation for maximum force production between the NuStep to the ID indicating that the individuals who had the capability to generate a greater force on the NuStep also produced greater force on the ID. Pending confirmation of these findings in a fully powered study, this could suggest the use of the NuStep as a modality to measure force production. Due to the closed kinematic chain setup, the device could be used to measure functional strength but does not have the ability to test a particular muscle group in isolation. (Figure 3).

This study highlights the importance of further testing the NuStep as a measure of endurance. Different methods of quadriceps muscle endurance exist in research and clinical practice. The ID, as mentioned previously, is used commonly in research but lacks a validated and standardized testing protocol. In addition, the ID equipment is expensive, can be time consuming to set up and intimidating for clinicians to use [19]. These drawbacks could limit its use in the clinic. Other easy to use and cheaper methods exist such as repeated SLR, repeated squats $[20,21]$ or wall squat holds [22]but these have yet to be scientifically validated [23]. In addition, similar to the ID, SLR is done in an open chain fashion, which may be less specific to daily functional demands and does not necessarily isolate a muscle or group of muscle. Clearly, endurance measures need to be investigated for feasibility, validity and reliability in order to assist clinicians in making clinical decisions regarding the most appropriate endurance test. The need for standardized measures of endurance in clinical settings has been expressed previously $[10,24]$. 
Valid clinical quadriceps endurance measures are important. Many patients encountered in the clinic have quadriceps endurance deficits that cannot solely be predicted based on strength measurements only [9]. Quadriceps endurance deficits may be the greatest contributor to task failure in patients with COPD and may be more closely related to task failure than strength deficits, cardiac, or ventilator limitations in this population [9]. Endurance testing is therefore proposed as a more sensitive measure of muscle dysfunction and functional limitation than strength in this population [24]. Endurance testing could also assist in defining more specific rehabilitation goals and be used as an outcome measure to monitor the effectiveness of both pharmacological and non-pharmacological interventions [10].

The NuStep is widely found in physical therapy clinics, is easy to set up and comfortable for patients to use. It was found to be a preferred mode of fitness equipment in older adults [25], found to be feasible and safe to use with adults with significant motor deficits[26,27] and has been recommended for exercise testing and intervention for older adults, those with neuromuscular impairments or those with balance deficits [28]. The new "NuStep Transit" shows promise for measuring quadriceps/proximal lower extremity muscle endurance, especially at 90 degrees of knee flexion. Moreover, it should be noted that most of the proximal lower extremity muscles, including the quadriceps, are key in providing stability during standing and walking; therefore, a decrease in ME in these muscles, could lead to decreased functional mobility. Further investigation using larger sample size including clinical population are needed before the NuStep can be used as a clinical measure of endurance.

\section{Conclusion}

In conclusion, this pilot study showed fair correlation between endurance measures on the NuStep and on the ID. Further studies are needed to validate the NuStep endurance protocol and to investigate the protocol in clinical population before it can be recommended as a clinical measure of endurance.

Acknowledgments: This study was supported by Wayne State University's Physical Therapy Department and Neuro-Tech Laboratory.

Competing interests: The authors declare that they have no competing interests.

\section{References}

1. Swain, D. and C. Brawner (2012). ACSM's Resource Manual for Guidelines for Exercise Testing and Prescription. Philadelphia, Lippincott Williams \& Wilkins.

2. Papa, E. V., H. Garg and L. E. Dibble (2015). "Acute effects of muscle fatigue on anticipatory and reactive postural control in older individuals: a systematic review of the evidence." Journal Geriatric Physical Therapy 38(1): 40-48.

3. Schwendner, K. I., A. E. Mikesky, W. S. Holt, Jr., M. Peacock and D. B. Burr (1997). "Differences in muscle endurance and recovery between fallers and nonfallers, and between young and older women." Journal Gerontology Series A Biological Sciences Medical Sciences 52(3): M155-160.

4. Vaara, J. P., H. Kyrolainen, J. Niemi, O. Ohrankammen, A. Hakkinen, S. Kocay and K. Hakkinen (2012). "Associations of maximal strength and muscular endurance test scores with cardiorespiratory fitness and body composition." Journal Strength Conditioning Research 26(8): 2078-2086.

5. Hopkinson, N. S., M. J. Dayer, S. Antoine-Jonville, E. B. Swallow, R. Porcher, A. Vazir, P. Poole-Wilson and M. I. Polkey (2013). "Central and peripheral quadriceps fatigue in congestive heart failure." International Journal Cardiology 167(6): 25942599.
6. Callaghan, M. J., C. J. McCarthy and J. A. Oldham (2001). "Electromyographic fatigue characteristics of the quadriceps in patellofemoral pain syndrome." Manual Therapy 6(1): 27-33.

7. Elboim-Gabyzon, M., N. Rozen and Y. Laufer (2013). "Quadriceps femoris muscle fatigue in patients with knee osteoarthritis." Clinical Interventions in Aging 8: 1071-1077.

8. Serres, I., V. Gautier, A. Varray and C. Prefaut (1998). "Impaired skeletal muscle endurance related to physical inactivity and altered lung function in COPD patients." Chest 113(4): 900-905.

9. Coronell, C., M. Orozco-Levi, R. Mendez, A. RamirezSarmiento, J. B. Galdiz and J. Gea (2004). "Relevance of assessing quadriceps endurance in patients with COPD." European Respiratory Journal 24(1): 129-136.

10. Maltais, F., M. Decramer, R. Casaburi, E. Barreiro, Y. Burelle, R. Debigare, P. N. Dekhuijzen, F. Franssen, G. Gayan-Ramirez, J. Gea, et al. (2014). "An official American Thoracic Society/ European Respiratory Society statement: update on limb muscle dysfunction in chronic obstructive pulmonary disease." American Journal Respiratory Critical Care Medicine 189(9): e15-62

11. Evans, R. A., E. Kaplovitch, M. K. Beauchamp, T. E. Dolmage, R. S. Goldstein, C. L. Gillies, D. Brooks and S. Mathur (2015). "Is Quadriceps Endurance Reduced in COPD? A Systematic Review." Chest 147(3): 673-684.

12. Nyberg, A., A. Tornberg and K. Wadell (2016). "Correlation between Limb Muscle Endurance, Strength, and Functional Capacity in People with Chronic Obstructive Pulmonary Disease." Physiotherapy Canada 68(1): 46-53.

13. Thornley, L. J., N. S. Maxwell and S. S. Cheung (2003). "Local tissue temperature effects on peak torque and muscular endurance during isometric knee extension." European Journal Applied Physiology 90(5-6): 588-594.

14. Portney, L. and M. Watkins (2009). Foundattions of Clinical Reserach: Applications to Practical. New York, NY, USA, Pearson.

15. Burdett, R. and J. Vanswearingen (1987). "Reliability of lsokinetic Muscle Endurance Tests." Journal Orthopedic Sports Physical Therapy 8(10): 484-488.

16. De Ruiter, C. J., M. I. P. Mallee, L. E. C. Leloup and A. De Haan (2014). "A Submaximal Test for the Assessment of Knee Extensor Endurance Capacity." Medicine and Science in Sports and Exercise 46(2): 398-406.

17. Saey, D. and T. Troosters (2008). "Measuring skeletal muscle strength and endurance, from bench to bedside." Clinical and Investigative Medicine 31(5): E307-E311.

18. White, C., K. Dixon, D. Samuel and M. Stokes (2013). "Handgrip and quadriceps muscle endurance testing in young adults." Springerplus 2.

19. Kisner, C and Colby, L. A, (2018). Therapeutic Exercises: foundations and techniques. Philadelphia, F.A. Davis.

20. Rissanen, A., H. Alaranta, P. Sainio and H. Harkonen (1994). "Isokinetic and Non-Dynamometric Tests in Low-Back-Pain Patients Related to Pain and Disability Index." Spine 19(17): 1963-1967.

21. LeFebvre, R. (2001). Low Back and Leg Endurance Tests. WSCC Clinics.

22. McIntosh, G., L. Wilson, M. Affleck and H. Hall (1998). "Trunk and lower extremity muscle endurance: normative data for adults." Journal of Rehabilitation Outomes Measurement 2(4): 20-39. 
23. Wilkerson, G. B., J. L. Giles and D. K. Seibel (2012). "Prediction of Core and Lower Extremity Strains and Sprains in Collegiate Football Players: A Preliminary Study." Journal of Athletic Training 47(3): 264-272.

24. Barreiro, E. and J. Gea (2015). "Respiratory and Limb Muscle Dysfunction in COPD." COPD 12(4): 413-426.

25. Looney, M. A. and J. H. Rimmer (2003). "Aerobic exercise equipment preferences among older adults: a preliminary investigation." Journal Applied Measurement 4(1): 43-58.

26. Billinger, S. A., J. K. Loudon and B. J. Gajewski (2008). "Validity of a Total Body Recumbent Stepper Exercise Test to Assess Cardiorespiratory Fitness." Journal of Strength and Conditioning Research 22(5): 1556-1562.
27. Peterson, M. D., L. Lukasik, T. Muth, P. Esposito, H. Haapala, P. M. Gordon, H. IglayReger and E. A. Hurvitz (2013). "Recumbent Cross-Training Is a Feasible and Safe Mode of Physical Activity for Significantly Motor-Impaired Adults With Cerebral Palsy." Archives of Physical Medicine and Rehabilitation 94(2): 401407.

28. Dalleck, L. C., E. C. Borresen, A. L. Parker, K. M. Abel, L. A. Habermann, S. J. McLaughlin and J. Tischendorf S. (2011). "Development of a metabolic equation for the NuStep recumbent stepper in older adults." Percept Mot Skills 112(1): 183-192. 